\title{
Tuberculosis in foreign students in Japan, 2010-2014: a comparison with the notification rates in their countries of origin
}

\author{
Masaki Ota, ${ }^{a}$ Kazuhiro Uchimura ${ }^{a}$ and Seiya Kato ${ }^{a}$ \\ Correspondence to Masaki Ota (email: otam@jata.or.jp).
}

This study characterizes the foreign students with tuberculosis (TB) registered in Japan from 2010 to 2014 and compares their TB notification rates with those in their countries of origin. The TB notification rates in foreign students were retrieved from the National Epidemiological Surveillance of Infectious Disease system in Japan. National TB notification data from 16 countries and areas were extracted from the World Health Organization's and the official health websites of the countries and areas.

There were 1128 foreign students in Japan who developed TB between 2010 and 2014; nearly half of the cases were from China ( $n=530,46.9 \%)$, and $688(61.0 \%)$ were male with a median age of 23 years. The TB notification data for foreign students were highest in students from the Philippines (675/100 000 person years, 95\% confidence interval: 372-977). The notification rates in foreign students from seven countries were significantly higher than the average notification rate in their countries of origin (China, Indonesia, Mongolia, Myanmar, Nepal, the Philippines and Viet Nam). The Republic of Korea and Taiwan, China had significantly lower rates in foreign students than in their countries of origin.

The notification rates for foreign students in Japan may reflect a more accurate risk of developing TB among the immigrants to Japan than the TB notification rates in their countries of origin. These results may be helpful to identify the immigrants' countries/areas of origin with the necessity of pre-entry TB screening.

J apan has successfully reduced the burden of tuberculosis (TB) in the past six decades from 590684 cases (698 per 100000 population) in 1951 to 19615 cases (15.4 per 100000 population) in 2014. ${ }^{1,2}$ However, imported TB from immigrants is still a challenge in eliminating TB in Japan. The proportion of immigrants among all TB cases in Japan has steadily increased from $2.4 \%$ in 2000 to $5.6 \%$ in 2014 . Immigrants accounted for $43 \%$ of the TB cases among those aged 20 to 29 years in 2014 . $^{1,3}$

The TB notification rates in foreign countries can be referenced for assessing the risk of TB importation from their immigrants. However, the estimate might not reflect the actual disease burden accurately. ${ }^{4-6}$ Calculating the notification rates for TB in immigrants to Japan by country of origin is a challenge since some cases are temporary visitors who cannot be distinguished in the record. Illegal and undocumented immigrants who do not count in the denominator for calculating the TB notification rates may lead to overestimating the TB burden. For most of the immigrant occupation designations, the total number of people engaged in that occupation (i.e. denominator) is not available.
To our best knowledge, it is only possible to determine the TB notification rate in foreign students in Japan because the National TB Surveillance System of Japan records the cases' occupations, and foreign students (student visa holders) are surveyed by the Japan Students Services Organization (JASSO) annually. ${ }^{7}$

This study characterizes the foreign students with TB registered from 2010 to 2014 and compares their notification rates with those in their countries of origin to investigate if there are discrepancies between them.

\section{METHODS}

\section{Study population}

This study aimed to include all foreign students in Japan. Both long-term and short-term (with a stay of less than one year) students were included. The study included students from 16 countries and areas: Bangladesh; Cambodia; China; Taiwan, China; India; Indonesia; Malaysia; Mongolia; Myanmar; Nepal; the Philippines; Republic of Korea; the Russian Federation; Sri Lanka; Thailand and Viet Nam. Other foreign countries were

\footnotetext{
Research Institute of Tuberculosis, Japan Anti-Tuberculosis Association, Kiyose, Tokyo, Japan. 
excluded as TB cases were less than four in the study period or the number of students from those countries was too small (less than 300 students) to be listed in the JASSO survey.

Foreign students studying at accredited educational institutions, including high schools, colleges/universities and vocational/professional schools, make up about $75 \%$ of foreign students in Japan. ${ }^{7}$ They undergo health screening at entry to the schools with chest X-rays as required by the School Health and Safety Act of $1958 .{ }^{8}$ The other $25 \%$ of foreign students, mostly entering unaccredited Japanese language schools, may or may not be offered health screening. Suspected TB cases are referred to nearby health facilities for TB laboratory confirmation testing (confirmed by sputum smear, TB culture, polymerase chain reaction and other identification tests such as a Capilia TB-Neo test [Tauns Co. Ltd., Izunokuni, Shizuoka, Japan] that are described elsewhere). ${ }^{9}$

\section{The National TB Surveillance System}

The National TB Surveillance System of Japan has been described elsewhere. ${ }^{10}$ Briefly, once a TB case is diagnosed, physicians are required by law to report the diagnosis to the local health department using a standard notification form. Collected data include the patient's identity, demographics, epidemiological, clinical and laboratory testing information. A public health nurse from the local health office interviews the case to obtain additional information (for example, occupation and details about the case contacts for contact investigation). Data are then deposited into the National Epidemiological Surveillance of Infectious Disease system of Japan (NESID). ${ }^{10}$ TB data of local and foreign students were retrieved from NESID for analysis.

\section{TB notification data among foreign students in Japan}

Information on age, sex, year of registration, mode of detection and country of origin of the TB cases, who were local and foreign students, were obtained from NESID. The notification rates for TB among foreign students were calculated as the number of TB cases registered in Japan from 2010 to 2014 divided by the number of foreign students in Japan at that period by country/area. The number of foreign students in Japan was obtained from JASSO's annual survey. ${ }^{7}$

\section{National TB notification data in foreign countries and areas}

The national TB notification data were obtained from the World Health Organization's website. ${ }^{2}$ TB notification data for Taiwan, China were obtained from the website of the Centers for Disease Control, Department of Health, Taiwan, China. ${ }^{11}$ Population estimate of different countries and areas were obtained from the United Nations Population Division's web site. ${ }^{12}$

\section{Statistical methods}

Descriptive analyses were done for the TB cases and notification rates in the foreign students and those of their countries of origin. The 95\% confidence intervals (Cl) were calculated by binomial test. Statistical analyses were conducted by $R$ software version 3.0.1 (R Foundation for Statistical Computing, Vienna, Austria).

\section{Ethics statement}

This is a review of routine TB surveillance data, and we obtained a waiver of ethical clearance from the institutional review board of the Research Institute of Tuberculosis, Tokyo, Japan (IRB\#: 27-16).

\section{RESULTS}

\section{Total TB cases}

In total, there were 1128 TB cases among the foreign students registered in Japan between 2010 and 2014; $688(61.0 \%)$ were males with a median age of 23 years. In comparison, among the Japanese students with TB $(n=872), 57.2 \%$ were males and the median age was 21 years old. The majority $(86.7 \%$ of the foreign students and $91.5 \%$ of the Japanese students) of the student TB cases were age 18 to 27 years. These cases were detected either through health screening at the educational institutions (57.3\% for foreign students and $45.1 \%$ for Japanese students) or during self-healthcare seeking at clinics and hospitals (42.7\% for foreign students and $54.9 \%$ for Japanese students) (Table 1 ). The foreign students with TB accounted for $56.4 \%$ of all the students with TB $(n=2000)$ and for $1.05 \%$ of all the TB cases $(n=107335)$ in Japan registered in the same period. The number of TB cases with known occupations was 104862 in that period. The majority of the foreign students with TB were from China 
Table 1. Number of TB cases in students, Japan, 2010-2014

\begin{tabular}{lrrrr}
\hline \multicolumn{1}{c}{ Characteristics } & \multicolumn{2}{c}{ Foreign students } & \multicolumn{2}{c}{ Local students } \\
& $n$ & $\%$ & \multicolumn{1}{c}{$\boldsymbol{n}$} & $\%$ \\
\hline Age (years) & & & & \\
$\quad$ 18-22 & 453 & 40.2 & 657 & 75.3 \\
$23-27$ & 525 & 46.5 & 141 & 16.2 \\
$\quad$ 27 & 150 & 13.3 & 74 & 8.5 \\
Sex & & & & \\
$\quad$ Male & 688 & 61.0 & 499 & 57.2 \\
$\quad$ Female & 440 & 39.0 & 373 & 42.8 \\
$\quad$ TB detection in Japan & & & & \\
$\quad$ Health screening & 646 & 57.3 & 393 & 45.1 \\
$\quad$ Self-health-care seeking at clinics and hospitals & 482 & 42.7 & 478 & 54.9 \\
Total & $\mathbf{1 1 2 8}$ & $\mathbf{1 0 0 . 0}$ & $\mathbf{8 7 2}$ & $\mathbf{1 0 0 . 0}$ \\
\hline
\end{tabular}

TB, tuberculosis.

(530 cases, 46.9\%) followed by Viet Nam (131 cases, $11.6 \%$ ) and Nepal (127 cases, 11.2\%) (Figure 1). Information on the countries of origin was unavailable in about $5 \%$ of the cases.

\section{TB notification rates}

The overall TB notification rates in foreign students are shown in Figure 2 with an increasing trend from 104 to 140 per 100000 population between 2011 and 2014. TB notification rates for foreign students were highest in students from the Philippines (675/100 000 person years [PYs], $95 \% \quad \mathrm{Cl}$ : 372-977/100 000 PYs) followed by Myanmar (617/100 000 PYs, 95\% Cl: 417-817/100 000 PYs) and Nepal (498/100 000 PYs, 95\% Cl: 385-611/100 000 PYs). TB notification rates in other countries were less than 400/100 000 PYs (Figure 3).

The TB notification rates in foreign students from seven countries were significantly higher than the average notification rate in their countries of origin: China (101 versus 65/100 000 PYs, Indonesia (361 versus 130/100 000 PYs), Mongolia (363 versus 166/100 000 PYs), Myanmar (617 versus 272/100 000 PYs), Nepal (498 versus 131/100 000 PYs), the Philippines (675 versus 234/100 000 PYs) and Viet Nam (231 versus 112/100 000 PYs). The notification rates in students from the Republic of Korea (38 versus 95/100 000 PYs) and Taiwan, China (27 versus $52 / 100000$ PYs) were significantly lower than that in their original countries and areas (Figure 3 ). In the other countries, the differences between the two notification rates were not statistically significant $(P>0.05)$.

\section{DISCUSSION}

To our knowledge, this is the first report on TB in an immigrant subpopulation in Japan. Almost all of the TB cases among foreign students were captured because most educational institutions in Japan conduct entry health screening with chest $X$-rays as required by law. Our results show that the overall TB notification rates in foreign students increased from 2010 to 2014. Increases in enrolment of foreign students from countries with higher notification rates of TB such as Viet Nam and Nepal in recent years may be the reason for the increase in the overall TB notification rate in foreign students in Japan. ${ }^{2,7}$

The national TB programmes of many countries might not capture all the TB cases either because of insufficient technical and managerial capacity or because TB patients seek health care from the private sector and are not reported to the programme ${ }^{4,5}$ unless special arrangements are made. ${ }^{6}$ Therefore their TB notification rates appear to be lower than in reality. This might explain the higher notification rates in foreign students than the overall TB notification rates in their original countries. This explanation is also supported by the findings of a previous study, which screened for TB in United States of America immigrants, that found a high prevalence of smear-negative TB (more than 500 per 100000 population) in those born in China, the Philippines and Viet Nam. ${ }^{13}$

Some of the student TB cases detected in Japan may not be incident cases but rather may be prevalent cases who had developed TB before they were screened 
Figure 1. Geographical distribution of countries and areas of origin of TB cases among foreign students who studied in Japan, 2010-2014*

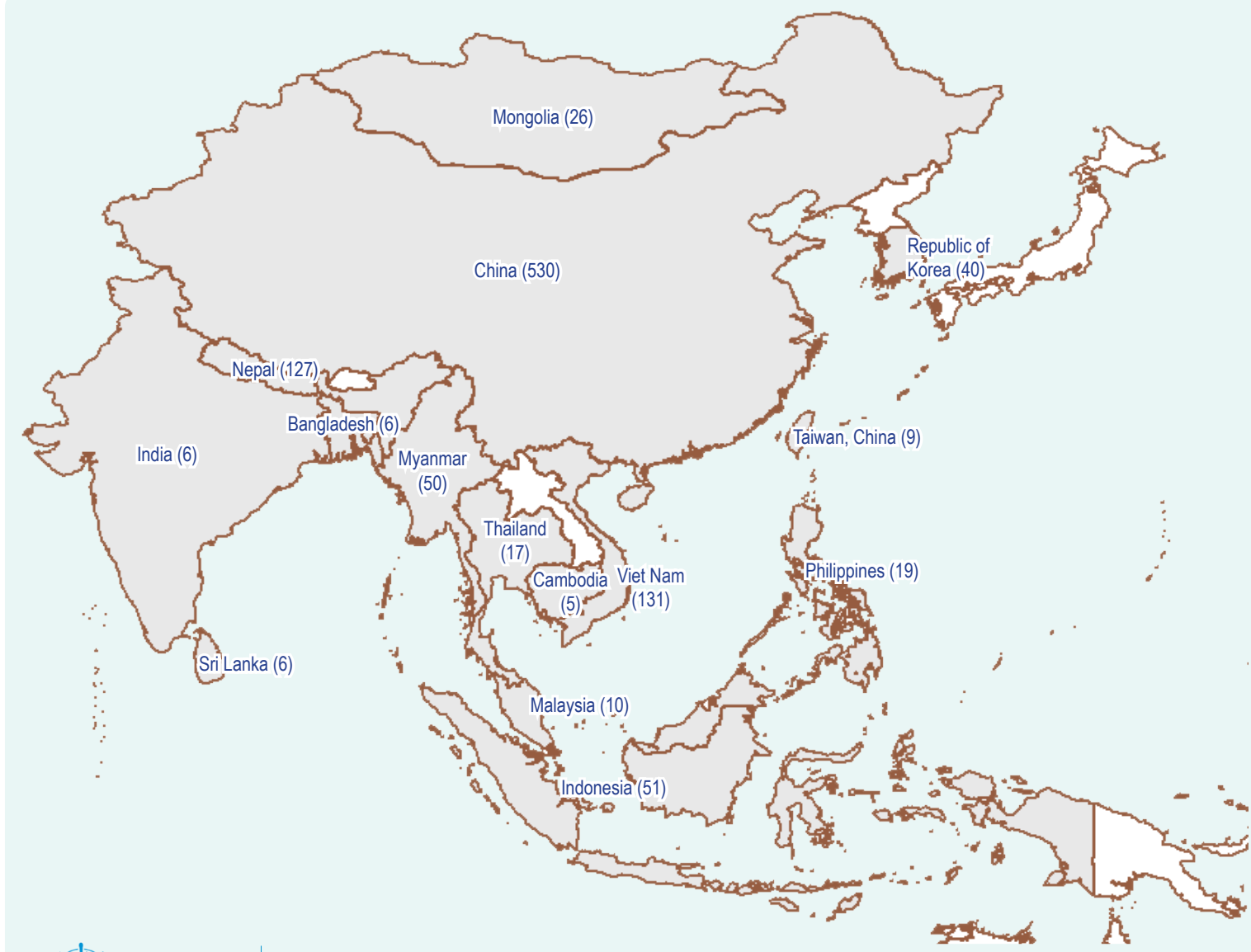

World Health Disclaimer: The boundaries shown and the designations used on this map do not imply the expression of any opinion 15 Organization whatsoever on the part of the World Health Organization concerning the legal status of any country, territory, city or area or of its authorities, or concerning the delimitation of its frontiers or boundaries. White lines on maps represent Western Pacific Region approximate border lines for which there may not yet be full agreement.

* Non-Asian countries and countries having fewer than four TB cases or fewer than 300 students are not shown.

TB, tuberculosis.

Figure 2. TB notification rates among foreign students, Japan, 2010-2014

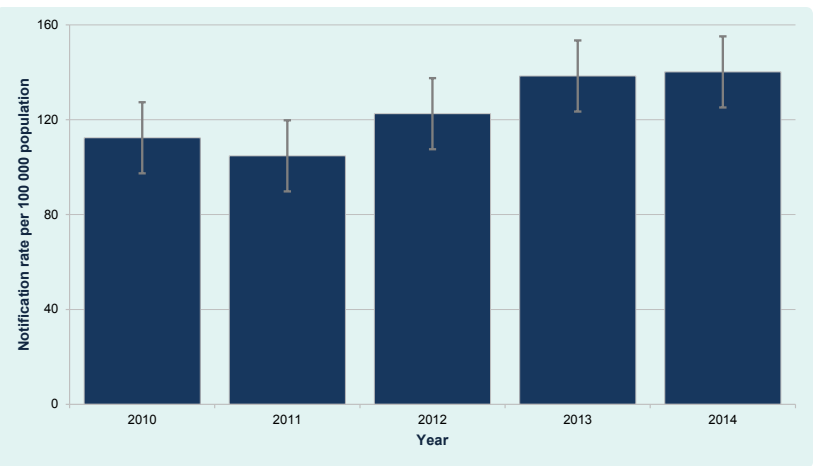

Error bars indicate $95 \%$ confidence intervals of the estimates. TB, tuberculosis. at the educational institutions. This might have made the student TB notification rates higher than the overall notification rate in their original countries and areas. However, about half of the TB patients sought health care only after they had arrived in Japan, suggesting they were incident cases. We believe the student TB notification rates mostly represent incidence of TB with some reflection of prevalence, although we do not know to what extent.

On the other hand, certain students may have come from regions with a higher TB notification rate than their national average. ${ }^{14}$ Also, students who had already developed TB may have transmitted the disease 
Figure 3. TB notification rates in foreign students in Japan and that of their countries and areas of origin, 2010-2014

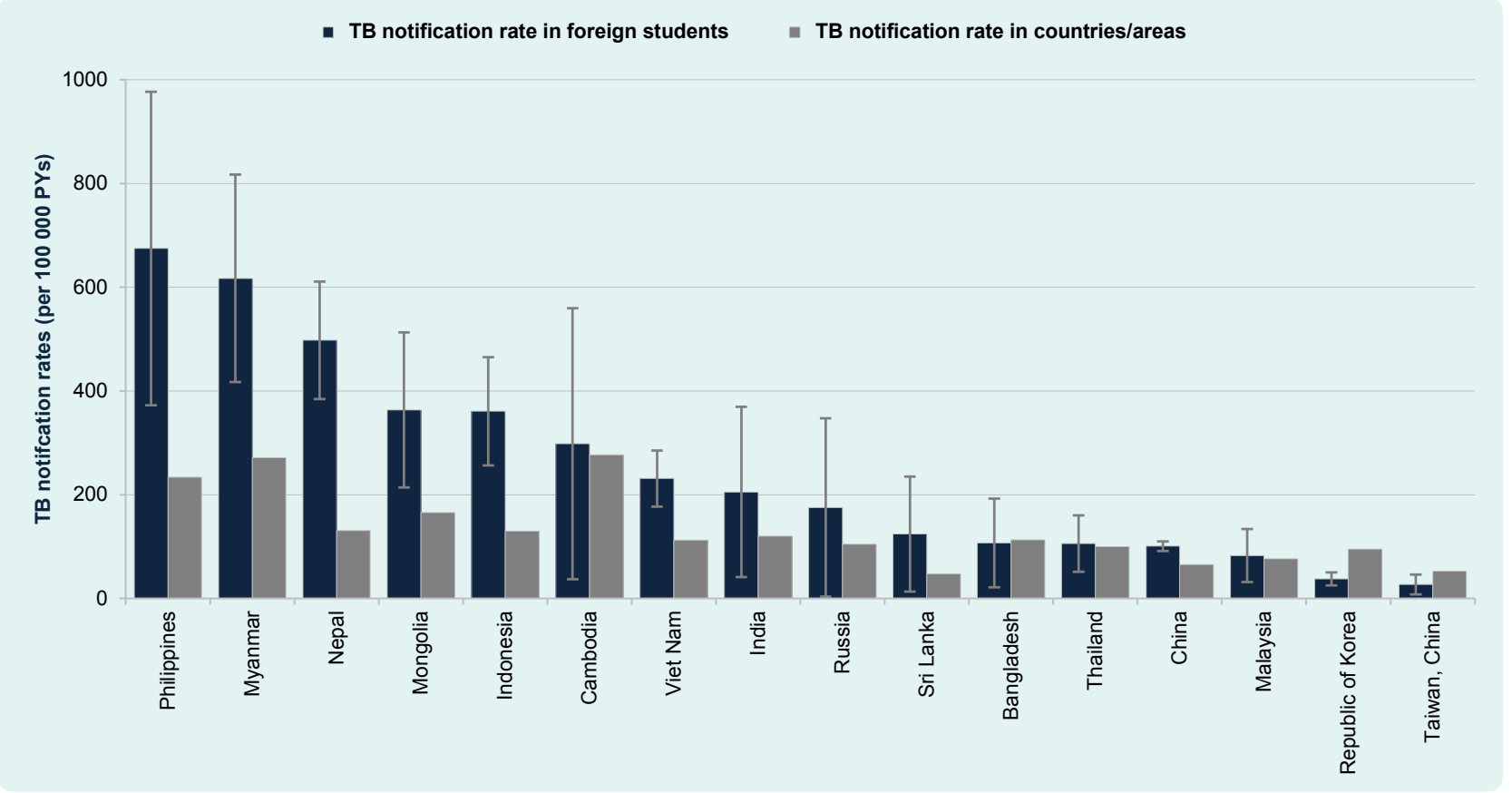

Error bars indicate $95 \%$ confidence intervals of the estimates.

PY, person years; TB, tuberculosis.

to other students after arriving in Japan. This could lead to a cluster of TB cases in the immigrant community and result in higher notification rates in this group than their national averages. In addition, in some countries and areas, TB burden is highest in the elderly population. Most of the foreign students from the Republic of Korea and Taiwan, China were aged 18 to 27 years, and the results show that their TB notification rates were lower than the national averages of their own countries and areas. $^{2,11}$

This study is based on routine surveillance data and has its limitations. First, the TB diagnostic data might have misclassifications or misdiagnoses. We were also unable to verify the occupational status data for each case. Second, about $5 \%$ of the foreign students with TB and information on their countries of origin were not available. The notification rates might be underestimated. Third, the survey conducted by JASSO might not capture all the students in Japan (nevertheless we were able to include short-term students who accounted for about $8 \%$ of the foreign students in this study). Furthermore, it is practically impossible to distinguish whether TB infections in the foreign students occurred before or after entering Japan. However, considering the low TB notification rate of Japanese residents, particularly young adults (less than 10 per 100000 population in 2014 for those aged 15 to 34 years) ${ }^{1}$ with whom foreign students are likely to socialize, the number of cases infected with TB after entering Japan is considered negligible.

We were unable to compare the case detection proportion between health screening at the education institutions (active screening) and self-health-care seeking at clinics and hospitals (passive screening) in this study as the total numbers of screened subjects in the two screening systems were not available. This should be addressed in future research.

Most countries with a low burden of TB in Europe, the Middle East, North America and Oceania have implemented systemic TB screening for immigrants, ${ }^{13,15}$ and such measures should be considered in Japan to further reduce the TB burden. ${ }^{16,17}$ In most South-East Asian countries, the peak age of developing TB is now shifting to the elderly (65 years or older). ${ }^{2}$ In assessing the risk of developing TB among immigrants who are mostly aged $20-50$ years, 
the TB notification rates in a subpopulation of immigrants may reflect the true TB rate better than the overall TB notification rate in their countries of origin especially when age-specific rates of these countries are not available. In future TB control programme management in Japan, the notification rates among foreign students can serve as a good reference to identify the immigrants' countries of origin with the necessity of pre-entry screening.

\section{Conflicts of interest}

None declared.

\section{Funding}

This study was supported through a Grant-in-Aid for Research Program on Emerging and Re-emerging Infectious Diseases (15fk0108004h001) by Japan Agency for Medical Research and Development and Ministry of Health, Labour, and Welfare.

\section{References}

1. Kekkaku no toukei 2015 (Statistics of TB 2015) [in Japanese]. Tokyo, Kekkaku Yobo kai (JATA), 2015.

2. Tuberculosis country profiles. Geneva, World Health Organization, 2015 (http://www.who.int/tb/country/data/profiles/en/, accessed 22 March 2016).

3. Tuberculosis Surveillance Center; RIT; JATA. [Tuberculosis annual report 2013-(1) Summary of tuberculosis notification statistics and foreign-born tuberculosis patients]. Kekkaku, 2015, 90:437443. pmid:26477115

4. Kumar M, Kumar S. Tuberculosis control in India: role of private doctors. Lancet, 1997, 350(9087):1329-1330. doi:10.1016/ S0140-6736(05)62497-X pmid:9357438

5. The burden of disease caused by TB. In: Global tuberculosis report 2014. Geneva, World Health Organization, 2014: 7-31 (http:// apps.who.int/iris/bitstream/10665/137094/1/9789241564809_ eng.pdf, accessed 22 March 2016).
6. Maung $\mathrm{M}$ et al. Private GPs contribute to TB control in Myanmar: evaluation of a PPM initiative in Mandalay Division. International Journal of Tuberculosis and Lung Diseases, 2006, 10(9):982987. pmid: 16964788

7. Result of an annual survey of international students in Japan, 2014 [in Japanese]. Tokyo, Japan Students Services Organization, 2014 (http://www.jasso.go.jp/about/statistics/intl_student_youryo/ index.html, accessed 22 March 2016).

8. Regulations related to the School Safety and Health Act of 1958 [in Japanese]. Tokyo, Ministry of Education, Culture, Sports, Science and Technology, 2008 (http://law.e-gov.go.jp/htmldata/ S33/S33F03501000018.html, accessed 22 March 2016).

9. National Standard of Tuberculosis Care (revised in 2016) [in Japanese]. Tokyo, Ministry of Health Labour and Welfare, 2016 (http://www.mhlw.go.jp/file/06-Seisakujouhou-10900000Kenkoukyoku/0000110838.pdf, accessed 22 March 2016).

10. Ohmori $\mathrm{M}$ et al. Computerized surveillance system of tuberculosis in Japan: its evolution, achievement and challenges. Kekkaku, 2012, 87(1):15-23. pmid:22416477

11. Centers for Disease Control annual report 2015. Taiwan, China, Ministry of Health and Welfare, 2015 (http://www.cdc gov.tw/uploads/files/201509/c9b61bbe-4a3b-40a9-8dceOf8b3d9cc2c7.pdf, accessed 22 March 2016).

12. Data query: Total population by sex (thousands). New York, United Nations Population Division, 2015 (http://esa.un.org/unpd/ wpp/DataQuery/, accessed 22 March 2016).

13. Liu $Y$ et al. Overseas screening for tuberculosis in U.S.-bound immigrants and refugees. New England Journal of Medicine, 2009, 360(23):2406-2415. doi:10.1056/NEJMoa0809497 pmid: 19494216

14. Wang $L$ et al. Tuberculosis prevalence in China, 1990-2010; a longitudinal analysis of national survey data. Lancet, 2014, 383(9934):2057-2064. doi:10.1016/S0140-6736(13)626392 pmid:24650955

15. Aldridge RW et al. Pre-entry screening programmes for tuberculosis in migrants to low-incidence countries: a systematic review and meta-analysis. Lancet Infectious Diseases, 2014 14(12):1240-1249. doi:10.1016/S1473-3099(14)70966-1 pmid:25455991

16. Ministry of Foreign Affairs et al. Stop TB Japan action plan. Tokyo, Stop TB Partnership Japan, 2014 (http:// www.stoptb.jp/dcms media/other/Action\%20PIan(F).pdf, accessed 5 April 2016).

17. Systematic screening for active tuberculosis: principles and recommendations. Geneva, World Health Organization, 2013 (http://www.who.int/tb/tbscreening/en/, accessed 22 March 2016). 